\title{
ÉVALUATION DE L'IMPACT SUR L'ÉCONOMIE RÉGIONALE DE LA PÊCHE AMATEUR : \\ L'EXEMPLE DES SALMONIDÉS MIGRATEURS.
}

\author{
F. BONNIEUX
}

INRA-Economie, rue Adolphe Bobierre, CS 61103, 35011 Rennes Cedex.

\section{RÉSUMÉ}

Cette contribution consacrée à une évaluation économique de la pêche au saumon et à la truite de mer s'appuie sur les résultats d'enquêtes de terrain réalisées tout au long de la saison 1990 et qui portent sur trois rivières de Basse-Normandie (Sée, Sélune et Touques) et une rivière de Bretagne (Elorn). Elles permettent de caractériser la population concernée de pêcheurs et leur pratique de pêche (expérience, effort) et d'évaluer les dépenses correspondantes.

Le refus de participer à l'enquête est demeuré exceptionnel puisqu'il n'y a eu que deux refus de la part d'individus suspectés de braconner. On dispose ainsi de 350 questionnaires exploitables et fiables comme le montrent différents rapprochements. II a été possible dans les trois-quarts des cas d'estimer le revenu. Les réponses fournies apparaissent sincères puisqu'elles sont cohérentes avec les données sur l'âge et la catégorie socioprofessionnelle. Ce sont les personnes les plus âgées souvent retraitées qui habitent à proximité du lieu de pêche qui ont tendance à refuser de répondre à cette question.

Ces enquêtes permettent tout d'abord de réaliser un portrait du pêcheur (âge, profession, revenu, lieu de résidence), de caractériser l'effort de pêche (matériel, nombre de visites, durée d'une visite) et la pratique (expérience, captures). De plus les informations recueillies sur les droits de pêche, les déplacements ef les dépenses d'hébergement et de nourriture aboutissent à une estimation moyenne du coût d'une saison de pêche aux salmonidés migrateurs qui atteint $6500 \mathrm{~F}$ (1990). Ce montant se décompose en $4400 \mathrm{~F}$ de frais de transport et de séjour, $1100 \mathrm{~F}$ de droits de pêche et $1000 \mathrm{~F}$ d'amortissement du matériel. Les pêcheurs résidant en Basse-Normandie dépensent nettement moins que les autres pêcheurs, leurs frais de transport et d'hébergement étant moins élevés. Par ailleurs, la pêche à la truite de mer est moins coûteuse que celle du saumon.

Les dépenses totales des pêcheurs aux salmonidés migrateurs qui ont péché en Basse-Normandie en 1990 se sont élevées à 17,5 millions de $F$ pour l'ensemble de la saison. Pour partie elles sont réalisées par des non-résidents et hors de la région. Tous calculs faits, il reste environ 14,2 millions de $F$ en Basse-Normandie dont 6,3 déboursés par des non-résidents qui correspondent donc à une exportation régionale. L'impact sur la valeur ajoutée régionale, qui constitue un indicateur de richesse, est de l'ordre de 10 millions de $F$ pour 1990. C'est une sous-estimation dans la mesure où une partie seulement des effets induits est prise en compte.

Mots-clés : coût d'une saison de pêche, effets économiques régionaux, saumon, truite de mer. 


\section{AN ECONOMIC ASSESSMENT OF RECREATIONAL ANGLING AT THE REGIONAL LEVEL : THE CASE-STUDY OF SEA TROUT AND SALMON.}

\section{ABSTRACT}

This contribution deals with an economic assessment of sea trout and salmon angling at the regional level. It relies on onsite surveys run during the 1990 fishing season and concerning four rivers, three in Lower-Normandy (Sée, Sélune and Touques) and one in Brittany (Elorn). They provide background information on anglers' characteristics, fishing experience and effort, as well as expenditures.

Only two sampled people refused to participate in the survey, both were suspected to be poachers. Finally there are 350 reliable questionnaires as different comparisons show and the household income has been estimated for $75 \%$ of the sample. Income estimation is valid, it is indeed consistent with occupation and age. Oldest people, often retired, do not will to give their income level.

Anglers' characteristics include age, occupation, income and home address. Fishing effort is described with the number and value of fishing rods and reels, the number of fishing days per year, and the length of a fishing day. Other indicators are related to fishing experience and catches. Otherwise information on fishing rights, travel costs, lodging and food expenditures result into an average cost of a fishing season equals to $1990 \mathrm{FF} 6,500$. This total amount includes, FF 1,100 of fishing rights, FF 4,400 of travel costs, lodging and food expenditures, and FF 1,000 of depreciation. The expenditures of anglers living in Lower-Normandy are under the average since they spend less for lodging and food. Otherwise, sea trout angling is less expensive than salmon angling.

Total expenditures of sea trout and salmon anglers who fished in Lower-Normandy have reached FF 17.5 millions for the 1990-fishing season. A certain share has been spending by non-residents and outside the region. The totall amount of money which benefited Lower-Normandy was FF 14.2 millions including FF 6.3 spent by non-residents which corresponds to a regional export. This results into an increase in regional value-added, which is an indicator of welfare, equals to FF 10 millions. Because all indirect effects are not taken into account, this figure under-estimates total impact on the regional economy.

Key-words : cost of a fishing season, regional economic impact, salmon, sea trout.

\section{INTRODUCTION}

La pèche est l'une des activitës de loisir de plein air les plus pratiquées en France ainsi que dans de nombreux autres pays. Les chiffres globaux sont trompeurs dans la mesure où il ne s'agit d'une pratique régullière que pour une minoritë de pêcheurs. Les données américaines qui sont parmi les plus complètes sur la question, montrent que le taux de participation des familles à revenu moven est nettement plus élevé que celui des familles à revenu faible, tandis qu"il diminue légèrement lorsque lion passe a la catégorie des revenus les plus élevés (WALSH, 1986). Cette baisse que ron constate aussi pour la chasse ne s'observe pas pour l'ensemble de loisirs de pleiln air, qunills alient un caractère sportif (bicyclette, jogging, équitation) ou quills soient étroitement liés à la nature (promenade, decouverte du millieu, observation des oiseaux, photographie de la faune). En faì la pêche de loisir est une catégorie composite qui agrège des activités diversifiées, parmi lesquelles certaines ont les caractéristiques d'un bien inférieur 
VERMERSCH, 1993). Les pêcheurs sont d'ailleurs capables de classer ces activités et situent la pêche sportive aux salmonidés migrateurs en haut de l'échelle (VAUGHAN et RUSSEL, 1982). II est intéressant de noter que les préférences des pêcheurs sont cohérentes avec une échelle synthétique de qualité de l'eau puisque la présence de truites et de saumons n'est possible que si un ensemble de paramètres de l'eau (acidité, concentration en oxygène dissous, température, particules en suspension) respecte des valeurs critiques.

Outre, une eau d'excellente qualité, la pêche aux salmonidés migrateurs exige beaucoup de temps de loisir et de technicité, et occasionne des dépenses élevées. C'est en ce sens un loisir haut de gamme qui assure une différentiation sociale marquée et ne concerne donc qu'une minorité de pêcheurs. On n'a compté en France en 1990 que 3000 pêcheurs de saumon et 3300 pêcheurs de truite de mer pour 1,9 million de permis délivrés. Au Canada dans l'ensemble formé par le Québec et les provinces maritimes où les ressources en saumon atlantique sont beaucoup plus abondantes, cette catégorie tout en étant beaucoup plus importante n'intéresse qu'un nombre relativement restreint de pêcheurs (122 000 sur près de 2 millions en 1985).

L'analyse présentée dans cette contribution est fondée sur les résultats d'enquêtes réalisées tout au long de la saison 1990 et selon la même méthode qui fait l'objet de la première section. Les principaux résultats de ces enquêtes sont ensuite rappelés dans les deux sections suivantes. La quatrième est consacrée à une évaluation des effets économiques agrégés (dépense et valeur ajoutée) à l'échelon de la Basse-Normandie.

\section{MÉTHODES}

En l'absence de toute information fiable sur les pêcheurs aux salmonidés migrateurs en France, on a procédé à des enquêtes de terrain pendant toute la durée de la saison de pêche 1990 (BONNIEUX et al., 1991). II s'agissait de caractériser cette population et sa pratique de pêche (expérience, effort), d'évaluer les dépenses correspondantes afin de disposer d'indicateurs de valeur économique de la pêche sportive. Les enquêtes ont porté sur trois rivières à saumon (l'Elorn située dans le Finistère, la Sée et la Sélune dans la Manche) et une rivière à truite de mer (la Touques située dans le Calvados).

Le travail des enquêteurs a été organisé selon un calendrier précis et un découpage des parcours de pêche. Lors de chaque vague d'enquêtes, les enquêteurs avaient pour mission d'interroger tous les pêcheurs rencontrés sur la section de rivière qui leur était attribuée. Ils ont pu ainsi interroger 173 pêcheurs de saumon (101 sur la Sée et la Sélune, et 72 sur l'Elorn) et 177 pêcheurs de truite de mer sur la Touques. Le calendrier des enquêtes intégrait l'information disponible sur le déroulement de la saison. Pour le saumon, on a la répartition suivante des questionnaires : $55 \%$ en mars, $35 \%$ en avril et $10 \%$ en mai et juin. Pour la truite de mer, on a : $17 \%$ en juin, $26 \%$ en juillet, $18 \%$ en août, $18 \%$ en septembre et $21 \%$ en octobre.

Le questionnaire identique pour l'ensemble des enquêtes porte sur les caractéristiques socioéconomiques des personnes interrogées, l'effort de pêche, la pratique de la pêche et les dépenses qui lui sont attachées. Le refus de participer à l'enquête est demeuré exceptionnel puisqu'il n'y a eu que deux refus de la part d'individus suspectés de braconner. L'interview prévue pour durer normalement 20 minutes a pris en moyenne une demi-heure, de nombreux pêcheurs souhaitant ajouter des commentaires personnels à leurs réponses. On dispose ainsi de 350 questionnaires exploitables et fiables comme le montrent différents rapprochements. II est toujours difficile d'évaluer le revenu des personnes interrogées dans un sondage. Dans cette enquête, les pêcheurs étaient invités à situer le revenu mensuel de leur famille sur une échelle distinguant 17 classes. Les refus de répondre ont été limités, eu égard à la nature de la question, 
puisqu'ils n'ont concerné en moyenne qu'un quart du total, avec un minimum de $17 \%$ pour l'Elorn et un maximum de $28 \%$ pour la Touques. Par ailleurs, 246 pêcheurs ont accepté de fournir leur adresse précise et ont reçu un compte-rendu d'enquête. Cette liste d'adresses a servi de base pour réaliser par la suite une enquête postale portant sur des points de gestion piscicole (BONNIEUX et al., 1992).

La représentativité des échantillons réunis lors d'enquêtes sur site est difficile à apprécier dans la mesure où il n'existe pas de base de sondage (POLLOCK et al, 1994). Bien que les timbres pour la pêche au saumon et à la truite de mer puissent être achetés sur l'ensemble du territoire, on a noté au cours des années quatre-vingts une forte concentration des achats dans les départements où sont situés les parcours et une quasi-absence de ventes en lle-de-France d'où viennent de nombreux pêcheurs (BONNIEUX et al,, 1991). Pour les timbres pour la pêche au saumon, une analyse statistique plus fine a pu être faite puisque l'origine géographique des acheteurs figure sur les bordereaux récapitulatifs. Les pêcheurs habitant la Basse-Normandie représentent $65 \%$ de l'échantillon interrogé sur la Sée et la Sélune et $68 \%$ des timbres achetés en Basse-Normandie en 1990. Les pêcheurs habitant le Finistère représentent $85 \%$ de l'échantillon interrogé sur l'Elorn et $86 \%$ des timbres vendus par l'association de l'Elorn en 1990. En retenant l'hypothèse d'un achat du timbre au voisinage du lieu de pêche on aboutit pour la Basse-Normandie à un nombre de pêcheurs de saumon compris entre 531 et 583 en 1990 selon que l'on inclut ou non les ventes réalisées en Ille-et-Vilaine. L'échantillon recueilli sur la Sée et la Sélune correspond donc à un taux de sondage de l'ordre de $18 \%$ de la population qui pêchait le saumon en Basse-Normandie en 1990. Appliquée aux pêcheurs de truite de mer, la même hypothèse conduit à un effectif total de 2214 individus pour la Basse-Normandie en 1990, ce qui correspond à un taux de sondage d'environ $8 \%$ pour l'échantillon recueilli sur la Touques.

\section{PORTRAIT DU PÊCHEUR}

\section{Caractéristiques des pêcheurs}

Les pêcheurs de salmonidés migrateurs constituent une population typée qui se distingue de la population française, comme le montre l'étude de variables socio-économiques classiques : sexe, âge, catégorie socioprofessionnelle et revenu. Par ailleurs la distinction entre pêcheurs locaux, habitant à proximité du lieu de pêche, et autres pêcheurs va fournir une grille de lecture utile puisque ces deux catégories présentent des différences socio-économiques significatives.

Le caractère fortement masculin de la pêche aux salmonidés migrateurs est confirmé par les trois échantillons, puisque trois femmes seulement ont pu être interrogées. La structure par classes d'âge fait ressortir la prédominance de la catégorie des 30-50 ans avec comme corollaire des effectifs relativement faibles, si on considère l'ensemble de la population française, de jeunes et dans une moindre mesure de plus âgés. Pour les plus âgés, il y a des différences selon les rivières, puisque ceux-ci sont fortement représentés sur la Sée et la Sélune, et beaucoup moins sur les deux autres rivières. Le calcul de l'âge moyen confirme cette spécificité de la Sée et de la Sélune puisqu'il atteint 46,8 ans contre 41,8 pour l'Elorn et 40,3 pour la Touques. Corrélativement on a $14 \%$ de retraités pour l'Elorn, $24 \%$ pour la Sée et la Sélune, et $10 \%$ pour la Touques, le chiffre étant de $14 \%$ pour la population masculine française. Si on raisonne maintenant sur les trois autres catégories socioprofessionnelles, il faut souligner la part élevée de cadres et techniciens pour les échantillons recueillis sur l'Elorn et la Touques et d'indépendants pour celui de la Sée et de la Sélune. A l'inverse, le nombre d'ouvriers et d'employés est faible pour la Sée et la Sélune et surtout pour l'Elorn, leur nombre se rapproche davantage de la moyenne nationale pour la Touques. 
La voiture est le moyen de transport privilégié pour se rendre sur les lieux de pêche puisqu'il concerne $96 \%$ des personnes interrogées, les écarts entre échantillons n'étant pas significatifs. Le partage d'un véhicule permet de réduire les coûts de transport et est plus fréquent sur la Touques, où il intéresse $52 \%$ des pêcheurs. Ce chiffre correspond soit à des pêcheurs voyageant ensemble, soit à des séjours familiaux sur place associés à l'expérience de pêche, qui sont plus fréquents sur cette rivière que sur les autres. A l'opposé le pourcentage plus élevé $(81 \%)$ de pêcheurs se déplaçant seuls dans le cas de l'Elorn correspond à l'origine plus locale que pour la Sée, la Sélune et la Touques. L'analyse de l'origine géographique des pêcheurs permet une description plus fine en tormes de clientèles. Elle souligne la prépondérance de la clientèle régionale qui représente de $63 \%$ pour la Touques à $88 \%$ du total pour l'Elorn. La distance moyenne parcourue entre le domicile et le lieu de pêche complète cette information. Comme on pouvait le prévoir, elle est plus faible avec $76 \mathrm{~km}$ pour l'Elorn, que pour les autres rivières puisque l'on trouve $85 \mathrm{~km}$ pour la Sée et la Sélune et $88 \mathrm{~km}$ pour la Touques. Le Tableau I précise ces données, la clientèle parcourant moins de $50 \mathrm{~km}$ et que nous qualifierons de locale représente en effet $62 \%$ des trois échantillons, avec un minimum de $55 \%$ pour la Touques et un maximum de $85 \%$ pour l'Elorn. Cette distance correspond à un déplacement de moins d'une heure, ce qui permet une fréquentation hebdomadaire et même pluri-hebdomadaire. Les pêcheurs qui parcourent des distances élevées constituent cependant un segment de clientèle non négligeable dans le cas des rivières normandes.

\section{Tableau I}

Distance du domicile au lieu de pêche.

Table 1

Trip distance from home to site.

\begin{tabular}{|c|c|c|c|c|c|c|c|c|}
\hline \multirow[b]{3}{*}{$\begin{array}{l}\text { Distance } \\
\text { Distance }\end{array}$} & \multicolumn{8}{|c|}{$\begin{array}{l}\text { Rivière } \\
\text { River }\end{array}$} \\
\hline & \multicolumn{2}{|c|}{ Sée-Sélune } & \multicolumn{2}{|c|}{ Elorn } & \multicolumn{2}{|c|}{ Touques } & \multicolumn{2}{|c|}{ Total } \\
\hline & $\begin{array}{l}\text { Nombre } \\
\text { Number }\end{array}$ & $\%$ & $\begin{array}{l}\text { Nombre } \\
\text { Number }\end{array}$ & $\%$ & $\begin{array}{l}\text { Nombre } \\
\text { Number }\end{array}$ & $\%$ & $\begin{array}{l}\text { Nombre } \\
\text { Number }\end{array}$ & $\%$ \\
\hline $\begin{array}{l}\text { Moins de } 10 \mathrm{~km} \\
\text { Less than } 10 \mathrm{~km}\end{array}$ & 30 & 29,7 & 38 & 52,8 & 20 & 11,3 & 88 & 25,2 \\
\hline $\begin{array}{l}10 \text { à } 50 \mathrm{~km} \\
\text { to to } 50 \mathrm{~km}\end{array}$ & 31 & 30,7 & 23 & 31,9 & 77 & 43,5 & 131 & 37,4 \\
\hline $\begin{array}{l}50 \text { a } 100 \mathrm{~km} \\
50 \text { to } 100 \mathrm{~km}\end{array}$ & 18 & 17,8 & 2 & 2,8 & 22 & 12,4 & 42 & 12,0 \\
\hline $\begin{array}{l}\text { Plus de } 100 \mathrm{~km} \\
\text { More than } 100 \mathrm{~km}\end{array}$ & 22 & 21,8 & 9 & 12,5 & 58 & 32,8 & 89 & 25,4 \\
\hline Total & 101 & 100,0 & 72 & 100,0 & 177 & 100,0 & 350 & 100,0 \\
\hline
\end{tabular}

Les résultats du Tableau II sont établis à partir d'un regroupement des données en quatre catégories de revenu. Les pêcheurs de salmonidés migrateurs se situent dans les tranches élevées de revenu, c'est-à-dire que par rapport à l'ensemble des ménages français, il y a un net décalage vers les catégories les plus aisées. En effet en 1988, $19 \%$ des ménages se situaient en dessous de $4500 \mathrm{~F}$ par mois et $34 \%$ en dessous de $6000 \mathrm{~F}$ alors que seulement $14 \%$ déclaraient plus de $15000 \mathrm{~F}$ et $6 \%$ plus de $20000 \mathrm{~F}$. En termes de revenu, cette population des pêcheurs apparaît donc relativement favorisée ce qui est cohérent avec la répartition par âge et par catégorie socioprofessionnelle. 
Les revenus élevés sont plus nombreux sur la Touques, où $7 \%$ des pêcheurs déclarent plus de $27000 \mathrm{~F}$ par mois, que sur les autres rivières. Le refus de répondre à la question sur le revenu est plus fréquent dans la population locale ainsi que chez les personnes les plus âgées qui sont souvent retraitées.

\section{Tableau II}

Revenu.

\section{Table II}

Income. FF : French Franc.

\begin{tabular}{|c|c|c|c|c|c|c|c|c|}
\hline & \multicolumn{7}{|c|}{$\begin{array}{c}\text { Rivière } \\
\text { River }\end{array}$} \\
\hline & \multicolumn{2}{|c|}{ Sée-Sélune } & \multicolumn{2}{|c|}{ Elorn } & \multicolumn{2}{|c|}{ Touques } & \multicolumn{2}{c|}{ Total } \\
\hline $\begin{array}{c}\text { Revenu (F 1990) } \\
\text { Income (FF 1990) }\end{array}$ & $\begin{array}{c}\text { Nombre } \\
\text { Number }\end{array}$ & $\%$ & $\begin{array}{l}\text { Nombre } \\
\text { Number }\end{array}$ & $\%$ & $\begin{array}{c}\text { Nombre } \\
\text { Number }\end{array}$ & $\%$ & $\begin{array}{c}\text { Nombre } \\
\text { Number }\end{array}$ & $\%$ \\
\hline $\begin{array}{c}\text { Moins de 6 100 F } \\
\text { Less than FF 6,700 }\end{array}$ & 22 & 26,2 & 12 & 22,6 & 28 & 22,0 & 62 & 23,5 \\
\hline $\begin{array}{c}6100-11700 \mathrm{~F} \\
\text { FF 6,100-11,700 }\end{array}$ & 27 & 32,1 & 17 & 32,1 & 31 & 24,4 & 75 & 28,4 \\
\hline $\begin{array}{c}11700-18 \text { 000 F } \\
\text { FF 11,700-18,000 }\end{array}$ & 21 & 25,0 & 11 & 20,8 & 38 & 29,9 & 70 & 26,5 \\
\hline $\begin{array}{c}\text { Plus de 18 000 F } \\
\text { More than FF 18,000 }\end{array}$ & 14 & 16,7 & 13 & 24,5 & 30 & 23,7 & 57 & 21,6 \\
\hline Total & 84 & 100,0 & 53 & 100,0 & 127 & 100,0 & 264 & 100,0 \\
\hline
\end{tabular}

\section{Effort et expérience}

L'effort de pêche est une notion composite, difficile à appréhender puisqu'll concerne l'ensemble des facteurs qui concourent à l'activité de pêche. Pour l'apprécier une batterie d'indicateurs qui décrivent le matériel de pêche, la durée de pêche et l'appât a été utilisée. Il faudrait pouvoir tenir compte aussi de l'habileté du pêcheur, variable bien évidemment difficile à mesurer. Des indicateurs comme le nombre de captures et le nombre d'années de pêche en rendent compte partiellement.

La mesure du matériel de pêche en quantité et en valeur ne soulève que des difficultés mineures. Les données sur le nombre de cannes et de moulinets montrent une différence significative entre l'Elorn et les rivières normandes (Tableau III).

\section{Tableau III}

Nombre de cannes et de moulinets (intervalles de confiance à 0,90 ).

Table III

Number of fishing rods and reels (90\% confidence intervals).

\begin{tabular}{|c|c|c|c|}
\hline & Sée-Sélune & Elorn & Touques \\
\hline $\begin{array}{c}\text { Cannes } \\
\text { Rods }\end{array}$ & $2,8 \pm 0,3$ & $5,6 \pm 1,1$ & $2,9 \pm 0,6$ \\
\hline $\begin{array}{c}\text { Moulinets } \\
\text { Reels }\end{array}$ & $3,2 \pm 0,4$ & $5,3 \pm 1,0$ & $2,3 \pm 0,6$ \\
\hline
\end{tabular}


La durée de pêche le jour de l'enquête est connue avec une bonne précision. Les pêcheurs ont en effet été interrogés sur leur heure d'arrivée sur la rivière ainsi que sur leur heure de départ. Pour cette dernière, il s'agit d'une prévision qui a pu être contrôlée dans quelques cas, l'enquêteur repassant en fin de journée. Connaissant ces deux valeurs et la durée des interruptions éventuelles en cours de journée, en particulier pour le déjeuner, le calcul est facile à mener à bien. De plus nous proposons une évaluation du temps consacré à la pêche sur l'ensemble de la saison et du nombre de visites (parties de pêche). Ces deux variables sont calculées à partir d'une discussion semi-directive entre le pêcheur et l'enquêteur, qui porte sur la pratique de la pêche d'une part depuis l'ouverture, d'autre part jusqu'à la fin de la saison. On se fonde donc sur un comportement effectif pour la première composante et un comportement envisagé pour la seconde. Cette approche apparaît a posteriori assez fiable compte tenu des pratiques effectives des pêcheurs de salmonidés migrateurs, marquées par la régularité et la ponctualité. C'est une pêche trop sérieuse pour déroger à ses plans que l'on soit en vacances pour une durée limitée ou que l'on vienne chaque fin de semaine. Cette approche qui ne serait sans doute pas applicable à d'autres contextes, aboutit à une évaluation de l'effort de pêche sur l'ensemble de la saison.

La durée de pêche le jour de l'enquête varie selon les rivières et les catégories de pêcheurs (Tableau IV). Notons tout d'abord l'opposition entre la Sée et la Sélune d'une part et la Touques d'autre part qui est facile à comprendre puisqu'il s'agit de pêches différentes impliquant des pratiques spécifiques. La pêche au saumon pratiquée sur la Sée et la Sélune se caractérise par une très longue présence au bord de la rivière alors que la pêche à la truite et en particulier à la truite de mer pratiquée sur la Touques se caractérise par des durées beaucoup plus courtes, c'est le « coup » du matin ou du soir. L'Elorn se situe en position intermédiaire, ce qui est normal puisque l'on a des pêcheurs de saumon et de truite. Les premiers représentent $70 \%$ de l'échantillon et les seconds $30 \%$ environ. On peut vérifier que compte tenu de ces coefficients, les durées moyennes obtenues sur l'Elorn sont égales à la moyenne pondérée des durées sur la Sée, la Sélune et la Touques, ce qui montre la cohérence des résultats. Les pêcheurs locaux passent moins de temps sur la rivière à chaque visite que les autres pêcheurs comme le montrent les écarts estimés entre les durées moyennes pour chaque cas. Ce résultat conduit à l'hypothèse d'une substitution entre durée de pêche et distance parcourue, plus on vient de loin plus on tient à rester longtemps au bord de la rivière. Pour terminer il faut noter que les durées de pêche observées le week-end de l'ouverture sont plus élevées que les autres jours, ce qui correspond à un comportement bien connu des pêcheurs.

\section{Tableau IV}

Durée de pêche le jour de l'enquête en heures (intervalles de confiance à 0,90).

\section{Table IV}

Average hours fished the day of the survey ( $90 \%$ confidence intervals).

\begin{tabular}{|c|c|c|c|}
\hline $\begin{array}{c}\text { Pêcheurs } \\
\text { Anglers }\end{array}$ & Sée-Sélune & Elorn & Touques \\
\hline $\begin{array}{c}\text { Locaux } \\
\text { Residents }\end{array}$ & $7,0 \pm 0,5$ & $6,1 \pm 0,6$ & $3,8 \pm 0,4$ \\
\hline $\begin{array}{c}\text { Autres } \\
\text { Non-residents }\end{array}$ & $9,8 \pm 0,3$ & $8,3 \pm 1,4$ & $6,0 \pm 0,7$ \\
\hline $\begin{array}{c}\text { Ensemble } \\
\text { Complete sample }\end{array}$ & $8,1 \pm 0,4$ & $6,5 \pm 0,6$ & $4,8 \pm 0,4$ \\
\hline
\end{tabular}


Lanalyse du nombre de visites pour l'ensemble de la saison (Tableau V) oppose une fois encore les locaux aux autres pêcheurs. Une analyse limitée aux pêcheurs habitant à moins de $10 \mathrm{~km}$ montrerait des fréquences encore plus élevées. Deux facteurs permettent de rendre compte des différences observées entre rivières. C'est tout d'abord le type de pêche pratiquée. Toutes choses égales par ailleurs, le saumon reste l'apanage de " mordus " qui sacrifient tous leurs autres loisirs à leur sport préféré. Le second facteur lié à la proximité du domicile explique la fréquence plus élevée des visites des locaux pour l'Elorn que pour les autres rivières.

\section{Tableau V}

Nombre de visites par pêcheur (ensemble de la saison).

Table V

Number of trips per angler during the fishing season.

\begin{tabular}{|c|c|c|c|}
\hline $\begin{array}{c}\text { Pêcheurs } \\
\text { Anglers }\end{array}$ & Sée-Sélune & Elorn & Touques \\
\hline $\begin{array}{c}\text { Locaux } \\
\text { Residents }\end{array}$ & 56 & 70 & 38 \\
\hline $\begin{array}{c}\text { Autres } \\
\text { Non-residents }\end{array}$ & 36 & 26 & 26 \\
\hline $\begin{array}{c}\text { Ensemble } \\
\text { Complete sample }\end{array}$ & 47 & 62 & 31 \\
\hline
\end{tabular}

La fréquence des visites et leur durée moyenne sont étroitement liées à la distance à parcourir. Les pêcheurs qui habitent à proximité viennent plus souvent et pour des durées plus courtes, ceci évidemment toutes choses égales par ailleurs et en particulier pour une même pêche. Ces substitutions correspondent à un comportement rationnel qui est conforme à la théorie du consommateur. La durée de la pêche sur l'ensemble de la saison est le produit d'une durée et d'une fréquence moyennes. Cette variable synthétique est donc la résultante de deux effets qui jouent en sens inverse lorsque la distance augmente : diminution du nombre de visites et accroissement de leur durée moyenne.

\section{Tableau VI}

Durée de pêche sur l'ensemble de la saison en heures (intervalles de confiance à 0,90).

\section{Table VI}

Annual hours fished $(90 \%$ confidence intervals).

\begin{tabular}{|c|c|c|c|}
\hline $\begin{array}{c}\text { Pêcheurs } \\
\text { Anglers }\end{array}$ & Sée-Sélune & Elorn & Touques \\
\hline $\begin{array}{c}\text { Locaux } \\
\text { Residents }\end{array}$ & $343 \pm 40$ & $304 \pm 26$ & $119 \pm 15$ \\
\hline $\begin{array}{c}\text { Autres } \\
\text { Non-residents }\end{array}$ & $308 \pm 43$ & $184 \pm 67$ & $117 \pm 19$ \\
\hline $\begin{array}{c}\text { Ensemble } \\
\text { Complete sample }\end{array}$ & $328 \pm 29$ & $284 \pm 25$ & $118 \pm 12$ \\
\hline
\end{tabular}


Les données du Tableau VI confirment que l'effort individuel sur la Touques est inférieur à celui observé sur les autres rivières, la durée de pêche sur l'ensemble de la saison est beaucoup plus faible que sur l'Elorn et plus encore que sur la Sée et la Sélune. Pour la Touques il n'y a pas d'écart entre pêcheurs locaux et autres pêcheurs : la fréquence plus faible des visites des premiers étant compensée par des durées moyennes plus longues par visite des seconds. La même observation vaut lorsque l'on considère les deux catégories de pêcheurs pour la Sée et la Sélune mais ne s'applique pas à l'Elorn. Pour cette dernière, rappelons la faible fréquence des visites des non - locaux, du même ordre que pour la Touques. Enfin, la durée pour les locaux est plus élevée sur la Sée et la Sélune que pour l'Elorn, l'impact durée moyenne dominant celui du nombre de visites.

L'expérience du pêcheur de truite de mer (mesurée en années) est inférieure à celle du pêcheur de saumon. On observe en moyenne une expérience de 7 ans sur la Touques, 15 ans sur la Sée et la Sélune et de 19 ans sur l'Elorn, ce qui correspond à un âge de début de 33 ans, 32 ans et 28 ans respectivement. Près de la moitié des personnes interrogées sur la Touques ont moins de 5 ans d'expérience. Cette catégorie de pêcheurs moins expérimentés est minoritaire sur les autres rivières, où au contraire la catégorie la plus nombreuse est celle de ceux qui pêchent depuis plus de 10 ans. Les 153 pêcheurs interrogés sur la Touques ont déclaré 540 captures, ce qui correspond à une moyenne annuelle en 1989 de 3,5 par personne. Mais le nombre de prises varie considérablement, puisque $48 \%$ des pêcheurs n'en déclarent aucune. La moyenne s'établit alors à 6,7 pour ceux qui ne sont pas revenus bredouilles et un effectif significatif déclare plus de 10 captures. Les captures déclarées de saumons pour les années 1987, 88 et 89 valent 225 pour la Sée et la Sélune et 223 pour l'Elorn, et ont été réalisées par 65 et 32 pêcheurs respectivement. Si on raisonne sur les seuls pêcheurs qui pratiquent depuis 1987 au moins, on obtient pour les trois années une moyenne de 3,8 saumons pour la Sée et la Sélune et de 7,3 pour l'Elorn, l'écart entre rivières étant significatif. On a donc une moyenne annuelle de 1,3 pour la Sée et la Sélune et de 2,4 pour l'Elorn. La dispersion du nombre de captures par pêcheur est importante, puisque l'on observe un maximum de 18 saumons et que $14 \%$ des pêcheurs n'ont capturé aucun saumon en trois saisons. Pour la truite de mer, il n'y a pas d'écart entre les pêcheurs locaux et les autres pêcheurs, en revanche, les pêcheurs locaux capturent davantage de saumons que les autres pêcheurs tant sur la Sée et la Sélune que sur l'Elorn.

Une relation positive est observée entre l'effort de pêche (exprimé en heures par saison) et les captures, ce qui est conforme à la logique. Mais l'influence de l'expérience de pêche est plus complexe. Une expérience minimale se révèle nécessaire pour réaliser des captures, les pêcheurs ayant moins de 5 ans d'expérience, ont donc moins de réussite que les autres. Mais l'effort a tendance à diminuer avec l'âge, ce qui explique une diminution des captures lorsque l'expérience dépasse certains seuils. Ainsi observe-t-on moins de captures dans la catégorie des pêcheurs de plus de 10 ans d'expérience que parmi ceux qui ont de 5 à 10 ans d'expérience.

\section{COÛT D'UNE SAISON DE PÊCHE}

Nous analysons tout d'abord les dépenses en droits de pêche, les dépenses liées au séjour (hébergement, nourriture) et au transport, enfin les dépenses en matériel de pêche. Les dépenses observées à l'occasion des enquêtes concernent un séjour particulier, le passage à la saison entière passe donc par la prise en compte du nombre de visites. Pour le poste transport, lorsque plusieurs personnes voyagent ensemble, on a retenu un coût moyen par personne. Dans plus de $90 \%$ des cas, les pêcheurs viennent uniquement pour pêcher ; imputer leurs dépenses à la seule pêche n'introduit donc pas de biais sensible. Les évaluations que nous proposons n'incluent pas les dépenses des personnes qui accompagnent le pêcheur, mais qui ne pêchent pas. Cette composante correspond à une fréquentation touristique liée à la pêche et est sans doute significative 
sur la Touques. Son évaluation supposerait des investigations supplémentaires que les enquêtes réalisées, ciblées sur la pêche, ne permettent pas.

Les droits de pêche incluent l'ensemble des cotisations à des AAPP, taxes et timbre ainsi que des locations éventuelles de droits et de parts de société. La répartition des droits payés en 1990 montre que si pour une large majorité, ils restent inférieurs à $1000 \mathrm{~F}$, pour une minorité importante, ils se situent bien au-dessus. L'adhésion à de multiples associations, en particulier sur la Sée et la Sélune explique la valeur élevée des droits payés en moyenne en 1990. Cette remarque vaut davantage pour les pêcheurs non-locaux (Tableau VII).

\section{Tableau VII}

Droits de pêche moyens (F 1990).

\section{Table VII}

Average fishing fees (FF 1990).

\begin{tabular}{|c|c|c|c|}
\hline $\begin{array}{c}\text { Pêcheurs } \\
\text { Anglers }\end{array}$ & Sée-Sélune & Elorn & Touques \\
\hline $\begin{array}{c}\text { Locaux } \\
\text { Residents }\end{array}$ & 944 & 807 & 642 \\
\hline $\begin{array}{c}\text { Autres } \\
\text { Non-residents }\end{array}$ & 2214 & 1383 & 1343 \\
\hline $\begin{array}{c}\text { Ensemble } \\
\text { Complete sample }\end{array}$ & 1447 & 895 & 959 \\
\hline
\end{tabular}

On a distingué les visites d'une journée (sans nuitée sur place) et les séjours de plus d'une journée avec hébergement sur place. La première catégorie est éclatée en trois sous-catégories selon que le déjeuner est pris sur place (pique-nique ou restaurant) ou à la maison, ce qui implique dans certains cas deux allers-retours du domicile au lieu de pêche si la partie de pêche s'étend sur toute la journée. Pour la deuxième catégorie, on oppose les hébergements marchands (hôtels, campings, gîtes et chambres d'hôtes) aux hébergements non-marchands (résidences secondaires, logements chez des parents ou des amis).

Les visites d'une journée intéressent près de trois pêcheurs sur quatre, avec des différences sensibles selon les rivières. Elles s'expliquent aisément par la distance à parcourir pour venir pêcher. Les pêcheurs qui habitent à proximité immédiate reviennent volontiers manger à la maison, la distance moyenne du domicile au lieu de pêche vaut alors $23 \mathrm{~km}$, ce qui correspond à une durée de transport aller-retour inférieure à une heure. Au-delà, on mange sur place, la distance depuis le domicile atteint alors $59 \mathrm{~km}$ d'où une durée aller-retour de l'ordre de deux heures par partie de pêche. Enfin, la visite avec hébergement intéresse les pêcheurs qui habitent relativement loin : de $171 \mathrm{~km}$ pour la Touques à $461 \mathrm{~km}$ pour l'Elorn.

Lorsque le pêcheur déjeune sur place, il préfère le pique-nique au restaurant. Celui-ci attire sensiblement plus d'individus sur la Sée et la Sélune que sur la Touques et l'Elorn. La faiblesse des effectifs ne permet pas une comparaison rigoureuse des structures d'hébergement selon les rivières. On peut noter cependant la part prépondérante des hébergements de type marchand, essentiellement l'hôtel et le camping, les autres formes (gîtes, chambres d'hôtes) n'ayant qu'une part marginale. La truite de mer 
correspond à une pêche estivale, ce qui explique la part plus élevée de l'hébergement en camping dans l'échantillon Touques. Dans l'ensemble, les séjours sont inférieurs à la semaine : c'est le cas de $84 \%$ d'entre eux. Le nombre de nuitées vaut alors en moyenne 2,7 puisqu'il y a une majorité de séjours de week-end. Les séjours d'une durée supérieure à la semaine sont rares, sauf sur la Touques où ils représentent $21 \%$ des séjours et correspondent à des vacances d'été. L'application de coûts unitaires débouche sur l'évaluation des dépenses moyennes de séjour et de transport (Tableau VIII).

\section{Tableau VIII}

Dépenses moyennes de séjour et de transport (F 1990).

\section{Table VIII}

Food, lodging and transportation average expenditures (FF 1990).

\begin{tabular}{|c|c|c|c|}
\hline $\begin{array}{c}\text { Pêcheurs } \\
\text { Anglers }\end{array}$ & Sée-Sélune & Elorn & Touques \\
\hline $\begin{array}{c}\text { Locaux } \\
\text { Residents }\end{array}$ & 3659 & 4118 & 1976 \\
\hline $\begin{array}{c}\text { Autres } \\
\text { Non-residents }\end{array}$ & 10527 & 14205 & 5949 \\
\hline $\begin{array}{c}\text { Ensemble } \\
\text { Complete sample }\end{array}$ & 6465 & 5852 & 3772 \\
\hline
\end{tabular}

L'écart entre pêcheurs locaux et autres pêcheurs s'explique par les différences de coût de transport et de type de séjour. En moyenne, les locaux habitent à environ $19 \mathrm{~km}$ de la rivière alors que les autres pêcheurs doivent parcourir plus de $190 \mathrm{~km}$, on a donc un rapport de 1 à 10. Les pêcheurs locaux ne sont intéressés que par des visites d'une journée, sans nuitée sur place. Sur l'Elorn, toutes les personnes qui passent au moins une nuit sur place habitent hors du Finistère, celle qui réside le plus près doit parcourir $230 \mathrm{~km}$. Pour la Sée et la Sélune, deux pêcheurs interrogés et séjournant sur place habitent la Manche, mais à au moins $65 \mathrm{~km}$. Même cas de figure sur la Touques, où si l'on compte trois habitants du Calvados parmi les personnes hébergées sur place, celle qui réside le plus près doit parcourir $60 \mathrm{~km}$ pour venir pêcher.

Les dépenses moyennes sur la Touques sont nettement inférieures à celles observées sur les autres rivières. Cet écart tient tout d'abord à un effort de pêche beaucoup plus faible en termes de durée et de nombre de visites. De plus, la partie de pêche à la truite de mer est plus courte que la partie de pêche au saumon. Les locaux que ce soit sur l'Elorn ou la Sée et la Sélune font couramment deux allers-retours dans la journée, lorsqu'ils reviennent déjeuner à la maison, alors que sur la Touques il y a un seul aller-retour pour cette catégorie de pêcheurs. Pour les non-locaux, le véhicule est plus souvent partagé sur la Touques, ce qui réduit les coûts de transport par personne.

La comparaison de la Sée et de la Sélune d'une part et de l'Elorn d'autre part ne fait apparaitre de différence sensible que pour les non-locaux. Elle s'explique en partie par l'éloignement du domicile. Cette catégorie de pêcheurs habite en moyenne à $188 \mathrm{~km}$ dans le cas de la Sée et de la Sélune, mais à $433 \mathrm{~km}$ dans celui de l'Elorn : chaque visite se traduit donc par une différence de coût de transport de l'ordre de $900 \mathrm{~F}$ auxquels il convient d'ajouter les autres dépenses. Le facteur distance rend donc compte en partie de la différence. Celle-ci doit cependant ètre interprétée avec précaution dans la mesure où les effectifs des catégories de pêcheurs sont limités, en particulier pour l'Elorn. 
La distribution de la valeur du matériel (Tableau IX) montre des différences importantes entre les pêcheurs sur l'Elorn, plus nombreux à se situer dans la strate plus de $10000 \mathrm{~F}$, et les autres pêcheurs. A cette opposition, s'ajoute celle qu'il y a lieu de faire entre locaux et non-locaux, les seconds possédant un matériel plus coûteux que les premiers. On connaît pour chaque pêcheur l'estimation de son matériel selon six tranches de valeur s'étalant de moins de $1000 \mathrm{~F}$ à plus de $20000 \mathrm{~F}$. L'évaluation de la valeur du matériel (moulinets, cannes et lignes) a été faite à partir de la médiane de chaque tranche et en retenant $25000 \mathrm{~F}$ pour la tranche supérieure. Dans la suite on a supposé qu'il était amorti sur une durée de cinq ans.

\section{Tableau IX}

Valeur du matériel (F 1990).

\section{Table IX}

Value of equipment (FF 1990).

\begin{tabular}{|c|c|c|c|c|c|c|}
\hline & \multicolumn{2}{|c|}{ Sée-Sélune } & \multicolumn{2}{c|}{ Elorn } & \multicolumn{2}{c|}{ Touques } \\
\hline $\begin{array}{c}\text { Valeur du matériel } \\
\text { Value of equipment }\end{array}$ & $\begin{array}{c}\text { Nombre } \\
\text { Number }\end{array}$ & $\%$ & $\begin{array}{c}\text { Nombre } \\
\text { Number }\end{array}$ & $\%$ & $\begin{array}{c}\text { Nombre } \\
\text { Number }\end{array}$ & $\%$ \\
\hline $\begin{array}{c}\text { Moins de 2 000 F } \\
\text { Less than FF 2,000 }\end{array}$ & 33 & 32,7 & 25 & 34,7 & 63 & 35,6 \\
\hline $\begin{array}{c}\text { 2 000 à 5 000 F } \\
\text { FF 2,000 - 5,000 }\end{array}$ & 35 & 34,6 & 21 & 29,2 & 64 & 36,1 \\
\hline $\begin{array}{c}5 \text { O000 à 10 000 F } \\
\text { FF 5,000- 10,000 }\end{array}$ & 19 & 18,8 & 12 & 16,7 & 27 & 15,3 \\
\hline $\begin{array}{c}\text { Plus de 10 000 F } \\
\text { More than FF 10,000 }\end{array}$ & 14 & 13,9 & 14 & 19,4 & 23 & 13,0 \\
\hline Total & 101 & 100,0 & 72 & 100,0 & 177 & 100,0 \\
\hline
\end{tabular}

Le coût élevé d'une saison de pêche sportive ressort des données moyennes du Tableau $X$. Lécart entre les deux catégories de pêche est dû aux différences d'effort de pêche, d'amortissement du matériel (cannes et moulinets) et de droits de pêche (le prix du timbre saumon est plus élevé que celui du timbre truite de mer). La distance moyenne parcourue par les pêcheurs est à l'origine de dépenses élevées. Elle est moyenne de $162 \mathrm{~km}$ pour le saumon et $176 \mathrm{~km}$ pour la truite de mer, valeurs proches de celles constatées dans des cas où la fréquentation est régulière au cours de la saison, on peut citer une moyenne de $158 \mathrm{~km}$ dans le Maryland aux États-Unis (WALSH, 1986). Le coût d'une saison de pêche est nettement inférieur pour les pêcheurs locaux que pour ceux qui habitent à plus de $50 \mathrm{~km}$ puisque, la moyenne s'établit respectivement à $4855 \mathrm{~F}$ et $9450 \mathrm{~F}$. Ces chiffres peuvent être rapprochés de ceux obtenus pour l'Angleterre et le Pays de Galles en 1988 pour les pêcheurs de salmonidés migrateurs (RADFORD et al., 1991). La moyenne actualisée s'établit pour 1990 à $4270 \mathrm{~F}$, mais il y a une très forte variabilité selon les régions (de $2960 \mathrm{~F}$ à $10460 \mathrm{~F}$ ) le pourcentage de non-résidents se situant entre $10 \%$ et $43 \%$ selon les cas. Le coût moyen d'une saison de pêche au saumon atlantique au Canada (TUOMI, 1987) atteindrait $8865 \mathrm{~F}$ aux prix de 1990, ce qui est très proche de la valeur que nous avons obtenue pour le saumon. A partir d'un estimation des effectifs de pêcheurs de saumon et de truite de mer, on a calculé un coût moyen pondéré de la pêche aux salmonidés migrateurs en Basse-Normandie. II vaut $6500 \mathrm{~F}$, et se décompose en $1100 \mathrm{~F}$ de droits de pêche, environ $1000 \mathrm{~F}$ d'amortissement de matériel et $4400 \mathrm{~F}$ de frais de séjour et de transport. 


\section{Tableau $X$}

Coût moyen d'une saison de pêche (F 1990). (1) : cotisations, parts de sociétés, timbres. (2) : Moulinets, cannes et lignes amortis sur cinq ans. (3) : coûts de transport, d'hébergement et de nourriture.

\section{Table $\mathrm{X}$}

Average cost of a fishing season (FF 1990). (1) : License, fishing society fees, special fees for salmon and sea trout. (2) : Depreciation of reels, rods and lines over a five-year period. (3) : Yearly expenditures in food, lodging and transportation.

\begin{tabular}{|c|c|c|}
\hline & $\begin{array}{c}\text { Saumon } \\
\text { Salmon }\end{array}$ & $\begin{array}{c}\text { Truite de mer } \\
\text { Sea-trout }\end{array}$ \\
\hline $\begin{array}{c}\text { Droits de pêche } \\
\text { Fishing fees }\end{array}$ & 1217 & 959 \\
\hline $\begin{array}{c}\text { Valeur du matériel } \\
\text { Value of equipment }\end{array}$ & 1112 & 1028 \\
\hline $\begin{array}{c}\text { Séjour et transport } \\
\text { Food, lodging and } \\
\text { transportation }\end{array}$ & 6215 & 3772 \\
\hline Total & 8544 & 5759 \\
\hline
\end{tabular}

\section{Tableau XI}

Dépenses totales des pêcheurs en 1990 (F).

\section{Table XI}

All anglers' expenditures in 1990 (FF).

\begin{tabular}{|c|c|c|c|c|c|c|}
\hline & \multicolumn{2}{|c|}{$\begin{array}{c}\text { Saumon } \\
\text { Salmon }\end{array}$} & \multicolumn{2}{c|}{$\begin{array}{c}\text { Truite de mer } \\
\text { Sea-trout }\end{array}$} & \multicolumn{2}{c|}{$\begin{array}{c}\text { Salmonidés migrateurs } \\
\text { Salmon and sea-trout }\end{array}$} \\
\hline $\begin{array}{c}\text { Dépenses } \\
\text { Expenditures }\end{array}$ & Total & $\begin{array}{c}\text { dont étranger } \\
\text { including } \\
\text { foreigners }\end{array}$ & Total & $\begin{array}{c}\text { dont étranger } \\
\text { including } \\
\text { foreigners }\end{array}$ & $\begin{array}{c}\text { Total } \\
\text { dont étranger } \\
\text { including } \\
\text { foreigners }\end{array}$ \\
\hline $\begin{array}{c}\text { Séjour } \\
\text { Food and } \\
\text { lodging }\end{array}$ & 1328144 & 869737 & 2617888 & 1833188 & 3946032 & 2702905 \\
\hline $\begin{array}{c}\text { Transports } \\
\text { Transportation }\end{array}$ & 1992150 & 1067844 & 5727150 & 2739675 & 7719300 & 3807519 \\
\hline $\begin{array}{c}\text { Droits de } \\
\text { péche } \\
\text { Fishing fees }\end{array}$ & 812172 & 446156 & 2122187 & 1222475 & 2934360 & 1668631 \\
\hline $\begin{array}{c}\text { Equipement } \\
\text { Equipment }\end{array}$ & 623889 & 339444 & 2261250 & 1107500 & 2885139 & 446944 \\
\hline Toial & 4756356 & 2723161 & 12728475 & 6902838 & 17484831 & 9625999 \\
\hline
\end{tabular}




\section{CALCUL DES EFFETS AGRÉGÉS}

Les dépenses totales des pêcheurs tant à l'intérieur qu'à l'extérieur de la région sont récapitulées dans le Tableau XI. Elles s'élèvent à 17,5 millions de francs : $73 \%$ concernent la truite de mer et $27 \%$ le saumon. Les pêcheurs qui ne résident pas en Basse-Normandie, dépensent 9,6 millions de francs soit $55 \%$ des dépenses totales.

Considérons maintenant les seules dépenses réalisées en Basse-Normandie. Les dépenses faites hors de la région concernent en premier lieu les non-résidents et le poste transport. Pour ce dernier, on a admis que les dépenses d'entretien des véhicules étaient effectuées près du domicile des pêcheurs. Par ailleurs, une partie des dépenses en matériel a été imputée hors de la région. Le Tableau XII présente le total des dépenses effectuées par les pêcheurs de toute origine en Basse-Normandie pour leur activité de pêche aux salmonidés migrateurs. Les dépenses totales effectuées dans la région s'élèvent à 14 millions de francs pour une année. 5,88 millions sont utilisés pour couvrir les frais de transport, soit $41 \%$ des dépenses totales. Le deuxième poste de dépense concerne les frais de séjour qui s'élèvent à 3,94 millions de francs et $28 \%$ du total. 2,93 millions servent à régler les droits de pêche et enfin 1,44 million sont des dépenses d'équipement.

\section{Tableau XII}

\section{Dépenses des pêcheurs effectuées en Basse-Normandie en 1990 (F).}

\section{Table XII}

Anglers' expenditures made in Lower-Normandy in 1990 (FF).

\begin{tabular}{|c|c|c|c|c|c|c|}
\hline & \multicolumn{2}{|c|}{$\begin{array}{c}\text { Saumon } \\
\text { Salmon }\end{array}$} & \multicolumn{2}{c|}{$\begin{array}{c}\text { Truite de mer } \\
\text { Sea-trout }\end{array}$} & \multicolumn{2}{c|}{$\begin{array}{c}\text { Salmonidés migrateurs } \\
\text { Salmon and sea-trout }\end{array}$} \\
\hline $\begin{array}{c}\text { Dépenses } \\
\text { Expenditures }\end{array}$ & Total & $\begin{array}{c}\text { dont étranger } \\
\text { including } \\
\text { foreigners }\end{array}$ & Total & $\begin{array}{c}\text { dont étranger } \\
\text { including } \\
\text { foreigners }\end{array}$ & $\begin{array}{c}\text { Total } \\
\text { dont étranger } \\
\text { including } \\
\text { foreigners }\end{array}$ \\
\hline $\begin{array}{c}\text { Séjour } \\
\text { Food and } \\
\text { lodging }\end{array}$ & 1328144 & 869737 & 2617888 & 1833188 & 3946032 & 2702905 \\
\hline $\begin{array}{c}\text { Transports } \\
\text { Transportation }\end{array}$ & 1518747 & 594442 & 4357313 & 1369838 & 5876060 & 1964280 \\
\hline $\begin{array}{c}\text { Droits de } \\
\text { pêche } \\
\text { Fishing fees }\end{array}$ & 812172 & 446156 & 2122187 & 1222475 & 2934360 & 1668631 \\
\hline $\begin{array}{c}\text { Equipement } \\
\text { Equipment }\end{array}$ & 284444 & 0 & 1158750 & 0 & 1438194 & 0 \\
\hline \begin{tabular}{c} 
Total \\
\hline
\end{tabular} & 3943508 & 1910314 & 10251138 & 4425500 & 14194646 & 6335816 \\
\hline
\end{tabular}

Les dépenses effectuées à l'occasion de la pêche au saumon ont rapporté presque 4 millions de francs et celles concernant la truite de mer un peu plus de 10 millions de francs. Ce sont donc $72 \%$ des dépenses qui sont effectuées à l'occasion de la pêche à la truite de mer. Mais proportionnellement les dépenses de transport et d'équipement bénéficiant à la région sont plus fortes pour la truite de mer, alors que les dépenses de séjour sont plus élevées pour le saumon. De la même manière les dépenses des étrangers à la région concernent proportionnellement plus les frais de séjour et les droits de pêche. 
L'ensemble des dépenses effectuées dans la région est à l'origine d'effets économiques induits indirects puisque toute dépense génère une augmentation de la valeur ajoutée et des consommations intermédiaires. L'application de multiplicateurs permet de calculer la valeur des effets économiques induits par la pêche sportive des salmonidés migrateurs en Basse-Normandie. Les calculs sont effectués à partir des données du tableau national des échanges interindustriels. En effet, il n'existe pas de données de comptabilité régionale suffisamment fines pour utiliser les seuls éléments régionaux. Les résultats auxquels on aboutit doivent être considérés comme des ordres de grandeur.

Les dépenses sont tout d'abord réparties entre les différentes branches de la comptabilité nationale:

- commerce (branches 57 - 64) : dépenses d'alimentation et achat d'essence et de matériel de pêche ;

- réparation et commerce de l'automobile ou garages (branche 65) : amortissement et entretien des véhicules; alimentation ;

- hôtels, cafés et restaurants ou HCR (branche 67) : frais de séjour hors

- services récréatifs, culturels et sportifs non-marchands (branche 96) : droits de pêche.

On obtient une nouvelle ventilation des dépenses par branche d'activité à laquelle on applique les coefficients techniques correspondants afin de déterminer l'effet sur la valeur ajoutée régionale (Tableau XIII). Les coefficients techniques sont donc des coefficients multiplicateurs. Pour chacune des branches, ils dépendent des rapports existant entre les consommations intermédiaires, l'investissement et le chiffre d'affaires réalisés.

\section{Tableau XIII}

Calcul de l'impact sur la valeur ajoutée régionale. (1) : les coefficients techniques sont ceux de 1987.

Table XIII

Determination of the effect on regional value-added. (1) : 1987 multipliers.

\begin{tabular}{|c|c|c|c|}
\hline $\begin{array}{l}\text { Branches } \\
\text { Activities }\end{array}$ & $\begin{array}{l}\text { Dépenses }\left(10^{6} \mathrm{~F}\right) \\
\text { Expenditures }\left(10^{6} \mathrm{~F}\right)\end{array}$ & $\begin{array}{l}\text { Coefficients } \\
\text { Multipliers }\end{array}$ & $\begin{array}{l}\text { Dépenses }\left(10^{6} \mathrm{~F}\right) \\
\text { Value-added }\left(10^{6} \mathrm{~F}\right)\end{array}$ \\
\hline $\begin{array}{c}\text { Commerces } \\
\text { Retail stores and gas }\end{array}$ & 5,72 & 0,79 & 4,52 \\
\hline $\begin{array}{c}\text { Garages } \\
\text { Car maintenance }\end{array}$ & 2,94 & 0,67 & 1,97 \\
\hline $\begin{array}{l}\text { HCR } \\
\text { Hotels and } \\
\text { restaurants }\end{array}$ & 2,61 & 0,63 & 1,64 \\
\hline $\begin{array}{l}\text { Services } \\
\text { Services }\end{array}$ & 2,93 & 0,65 & 1,90 \\
\hline Total & 14,20 & & 10,03 \\
\hline
\end{tabular}


Toutes les branches concernées sont productrices d'une forte valeur ajoutée, ce qui entraîne une création relativement importante de richesse dans la région que l'on peut évaluer à environ 10 millions de francs pour 1990. II s'agit d'une sous-estimation puisque l'on ne tient pas compte ni des effets induits par cette richesse supplémentaire, ni de ceux qui résultent de l'augmentation corrélative des consommations intermédiaires, de l'investissement et du commerce extérieur de la région.

\section{CONCLUSION}

Le pêcheur moyen de salmonidés migrateurs est un quadragénaire qui appartient à un milieu social assez favorisé et dispose donc d'un revenu supérieur à la moyenne. Ce profil moyen, certes utile pour disposer d'un repère, est cependant trompeur car la population des pêcheurs n'est évidemment pas homogène. Ainsi, rencontre-t-on de nombreux retraités sur la Sée et la Sélune et un groupe important d'ouvriers sur la Touques. II n'empêche que l'on doit souligner la prédominance relative, c'est-à-dire par rapport à la population française, de la catégorie constituée par les cadres, les techniciens et les professions libérales. Cette prédominance est beaucoup plus marquée chez les pêcheurs qui viennent de loin, que parmi les pêcheurs locaux qui appartiennent dans l'ensemble à des milieux sociaux plus populaires.

Les pêcheurs possèdent un matériel varié qu'ils utilisent de façon intensive comme le montrent les estimations de durée de pêche par journée et sur l'ensemble de la saison. Ici il convient toutefois d'opposer la pêche au saumon et la pêche à la truite de mer. La première est beaucoup plus exigeante en temps et suppose de longs séjours au bord de la rivière, avec adaptation au cours de la journée au comportement du poisson d'où utilisation successive de différents appâts. Pour la seconde on a souvent des parties de pêche courtes qui s'inscrivent dans une demi-journée alors que pour le saumon la norme est plutôt la journée. Ces comportements tranchés entraînent des différences sensibles d'effort de pêche sur l'ensemble de la saison. Globalement le total des captures est élevé, mais ramené au pêcheur les résultats sont plus modestes : 3,5 truites de mer par saison et 3,8 saumons en trois saisons. Mais les moyennes ont une signification limitée compte tenu de la forte variabilité des captures et du nombre élevé de pêcheurs qui n'ont rien attrapé. Ce qui est intéressant de souligner ici c'est la plus grande réussite des pêcheurs locaux ainsi qu'une relation positive entre effort de pêche et captures. De plus un apprentissage de quelques années se révèle nécessaire sans que ce soit un gage de réussite.

L'analyse des résultats d'enquête introduit donc une distinction entre une clientèle locale et une clientèle qui habite beaucoup plus loin du lieu de pêche. On retrouve icila distinction classique qui est souvent faite entre les résidents (pêcheurs locaux) et les touristes. Ces deux catégories se distinguent par leur mode de fréquentation de la rivière. On observe chez les premiers de nombreuses visites d'une journée et même moins, régulièrement sur l'ensemble de la saison, chez les seconds il s'agit plutôt de séjours de plusieurs jours (type week-end) renouvelés ou de séjours en famille pendant la période estivale. C'est ici la durée du déplacement et son coût qui expliquent ces deux catégories de comportement. Les coûts correspondants sont très différents puisque pour les résidents les dépenses portent essentiellement sur l'achat de matériel, le paiement de droits de pêche et le transport. Pour les autres pêcheurs, il convient d'ajouter les dépenses supplémentaires occasionnées par le séjour (hébergement et nourriture).

Toutes catégories de pêcheurs et de dépenses confondues on peut estimer le coût d'une saison de pêche aux salmonidés migrateurs à environ $6500 \mathrm{~F}$ qui se décompose en $4400 \mathrm{~F}$ de frais de séjour et de transport, $1100 \mathrm{~F}$ de droits de pêche et $1000 \mathrm{~F}$ d'amortissement du matériel. Les résidents dépensent cependant nettement moins que les autres et la pêche à la truite de mer est moins coûteuse que celle au saumon. On a pu estimer les dépenses totales des pêcheurs aux salmonidés migrateurs qui ont pêché en 
Basse-Normandie en 1990. Elles s'élèvent à 17,5 millions de F pour l'ensemble de la saison. Pour partie ces dépenses sont faites par des étrangers à la Basse-Normandie et hors de la région. Tous calculs faits, il reste environ 14,2 millions de $F$ en BasseNormandie dont 6,3 déboursés par des non-résidents qui correspondent donc à une exportation ce qui améliore le solde du commerce extérieur de la région. Le calcul économique peut être poussé au-delà de ces évaluations et intégrer les effets induits sur la valeur ajoutée régionale qui constitue un indicateur de richesse. II a pu être estimé à au moins 10 millions de $\mathrm{F}$ en 1990. II s'agit sans doute d'une sous-estimation parce que tout d'abord ne sont pris en compte qu'une partie des effets induits. De plus, les calculs n'intègrent que les dépenses de séjour des pêcheurs, or en période estivale, ils sont souvent accompagnés de leur famille d'où des dépenses liées à la pêche.

\section{BILIOGRAPHIE}

BONNIEUX F., BOUDE J.P., GUERRIER C., RICHARD A., 1991. La pêche sportive du saumon et de la truite de mer en Basse-Normandie. Rapport CSP-INRA-ENSAR, $78 \mathrm{p}$.

BONNIEUX F., DESAIGUES D., VERMERSCH D., 1992. France. In : NAVRUD S., Pricing the European environment, Scandinavian University Press, Oslo, 45-64.

BONNIEUX F., VERMERSCH D., 1993. Bénéfices et coûts de la protection de l'eau. Revue d'Economie Politique, 103, 131-152.

POLLOCK K.H., JONES C.M., BROWN T.L., 1994. Angler survey methods and their applications in fisheries management. American Fisheries Society, Bethesda, Maryland, $369 \mathrm{p}$.

RADFORD A.F., HATCHER A.C., WHITMARSH D.J., 1991. An economic evaluation of salmon fisheries in Great Britain. Report prepared for the Ministry of Agriculture, Fisheries and Food, London, $334 \mathrm{p}$.

TUOMI A.L.W., 1987. Canada's Atlantic salmon recreational fisheries and their future, an economic overview. Report for the Atlantic Salmon Federation, St Andrews, N.B. Canada, $29 \mathrm{p}$.

VAUGHAN W.J., RUSSEL C.S., 1982. Freshwater recreational fishing, the national benefits of water pollution control. Resources for the Future, Washington D.C., $205 \mathrm{p}$.

WALSH R.G., 1986. Recreation economic decisions, comparing benefits and costs. Venture Publishing Inc. State College, Pennsylvania, 638 p. 